\title{
Fitting neutrino masses in a realistic intersecting D-braneworld
}

\author{
Jordan Gemmill, Evan Howington $\odot$, and Van E. Mayes \\ Department of Physical and Applied Sciences, University of Houston-Clear Lake, \\ Houston, Texas 77058, USA
}

(Received 20 August 2019; published 27 December 2019)

\begin{abstract}
The correct quark and charged lepton mass matrices along with a nearly correct Cabibbo-KobayashiMaskawa (CKM) quark mixing matrix may be naturally accommodated in a Pati-Salam model constructed from intersecting D6 branes on a $T^{6} /\left(\mathbb{Z}_{2} \times \mathbb{Z}_{2}\right)$ orientifold. Furthermore, near-tribimaximal mixing for neutrinos may arise naturally due to the structure of the Yukawa matrices. Consistency with the quark and charged lepton mass matrices in combination with obtaining near-tribimaximal mixing fixes the Dirac neutrino mass matrix completely. Then, applying the seesaw mechanism for different choices of righthanded neutrino masses and running the obtained neutrino parameters down to the electroweak scale via the renormalization group equations, we are able to make predictions for the neutrino masses and mixing angles. We obtain lepton mixing angles which are close to the observed values, $\theta_{12}=33.8^{\circ} \pm 1.2^{\circ}$, $\theta_{23}=46.9^{\circ} \pm 0.9^{\circ}$, and $\theta_{13}=8.56^{\circ} \pm 0.20^{\circ}$. In addition, the neutrino mass-squared differences are found to be $\Delta m_{32}^{2}=0.0025 \pm 0.0001 \mathrm{eV}^{2}$ and $\Delta m_{21}^{2}=0.000075 \pm 0.000003 \mathrm{eV}$ with $m_{1}=0.0150 \pm$ $0.0002 \mathrm{eV}, \quad m_{2}=0.0173 \pm 0.0002 \mathrm{eV}, \quad$ and $\quad m_{3}=0.053 \pm 0.002 \mathrm{eV} \quad$ so that $\sum_{i} m_{i}=0.085 \pm$ $0.002 \mathrm{eV}$, consistent with experimental observations.
\end{abstract}

DOI: 10.1103/PhysRevD.100.115048

\section{INTRODUCTION}

One of the most significant challenges in high-energy physics today is to explain the pattern of masses and mixing angles exhibited by the elementary fermions in the Standard Model (SM). In the cases of the quarks and charged leptons, the masses are strongly hierarchical, while the masses of the neutrinos are known to be very small in comparison. In addition, the quark mixing angles are relatively small while in contrast some of the mixing angles for the neutrinos are quite large. An explanation for the differences in masses and mixing angles between the neutrinos and quarks is currently somewhat of a mystery, though the seesaw mechanism does provide a way to obtain naturally small neutrino masses.

There has been some progress toward understanding the origin of the quark and lepton masses and mixing angles by extending the SM to include discrete flavor symmetries. Indeed, one of the most promising such discrete symmetries is $\boldsymbol{\Delta}(\mathbf{2 7})$. With this discrete symmetry, it has been shown that it is possible to explain the masses and mixing angles for quarks and leptons [1]. In particular, $\Delta(27)$ contains $\mathbf{A 4}$ as a subgroup, and it is known that mass matrices resulting from imposing an $\mathbf{A 4}$ symmetry naturally leads to tribimaximal

Published by the American Physical Society under the terms of the Creative Commons Attribution 4.0 International license. Further distribution of this work must maintain attribution to the author(s) and the published article's title, journal citation, and DOI. Funded by SCOAP . mixing, which may be taken as a zeroth-order mixing for the neutrinos. Although it might be possible to completely understand the origin of quark and lepton mixing by imposing such discrete flavor symmetries, this is still somewhat ad hoc. The actual origin of these symmetries remains unexplained. In fact, ultimately it should be possible to trace the origin of these discrete flavor symmetries back to some fundamental theory. String theory is a leading candidate for such a theory.

Recently, it has been shown in a particular string model constructed in Type IIA string theory with intersecting Dbranes that the mass matrices for the quarks and leptons are the same as those which are obtained by imposing a $\Delta(\mathbf{2 7})$ flavor symmetry [2]. Furthermore, it was demonstrated that it is possible to obtain mass matrices for the quarks and charged leptons which results in the correct masses as well as the correct Cabibbo-Kobayashi-Maskawa quark mixing matrix, or CKM matrix. In addition, it is also possible to simultaneously obtain a Dirac mass matrix for the neutrinos which results in tribimaximal mixing. Our approach then was to use the known masses for the quarks and charged leptons as inputs, as well as the tribimaximal constraint in order to completely determine the Dirac neutrino mass matrix. These results are highly nontrivial as the mass matrices for quarks and leptons in the model are not independent.

Although the results for the neutrinos give the correct mass-squared differences and the correct mixing matrix, these results are calculated at the string scale, which in the following we take to be the standard grand unified theory 
(GUT) scale, $M_{\mathrm{GUT}}=2 \times 10^{16} \mathrm{GeV}$. In order to make a valid comparison with experimental observations, it is necessary to evolve the neutrino mass parameters down to the electroweak scale, $M_{\mathrm{EW}}=100 \mathrm{GeV}$. In the following, we perform a renormalization group equation (RGE) analysis using the REAP 11.4 Mathematica package $[3,4]$. We obtain lepton mixing angles which are close to the observed values, $\theta_{12}=33.8^{\circ} \pm 1.2^{\circ}, \theta_{23}=46.9^{\circ} \pm 0.9^{\circ}$, and $\theta_{13}=8.56^{\circ} \pm 0.20^{\circ}$. In addition, the neutrino masssquared differences are found to be $\Delta m_{32}^{2}=0.0025 \pm$ $0.0001 \mathrm{eV}^{2}$ and $\Delta m_{21}^{2}=0.000075 \pm 0.000003 \mathrm{eV}^{2}$ with $m_{1}=0.0150 \pm 0.0002 \mathrm{eV}, \quad m_{2}=0.0173 \pm 0.0002 \mathrm{eV}$, and $m_{3}=0.053 \pm 0.002 \mathrm{eV}$ so that $\sum_{i} m_{i}=0.085 \pm$ $0.002 \mathrm{eV}$, consistent with experimental observations.

\section{A REALISTIC INTERSECTING D-BRANE MODEL}

In recent years, intersecting D-brane models have provided an exciting approach toward constructing semirealistic vacua. To summarize, D6 branes (in Type IIA) fill three-dimensional Minkowski space and wrap 3-cycles in the compactified manifold, with a stack of $N$ branes having a gauge group $U(N)$ [or $U(N / 2)$ in the case of $\left.T^{6} /\left(\mathbb{Z}_{2} \times \mathbb{Z}_{2}\right)\right]$ in its world volume. The 3-cycles wrapped by the D-branes will in general intersect multiple times in

TABLE I. General spectrum for intersecting D6-branes at generic angles, where $I_{a a^{\prime}}=-2^{3-k} \prod_{i=1}^{3}\left(n_{a}^{i} l_{a}^{i}\right)$ and $I_{a 06}=$ $2^{3-k}\left(-l_{a}^{1} l_{a}^{2} l_{a}^{3}+l_{a}^{1} n_{a}^{2} n_{a}^{3}+n_{a}^{1} l_{a}^{2} n_{a}^{3}+n_{a}^{1} n_{a}^{2} l_{a}^{3}\right)$. Moreover, $\mathcal{M}$ is the multiplicity, and $a_{S}$ and $a_{A}$ denote the symmetric and antisymmetric representations of $U\left(N_{a} / 2\right)$, respectively.

\begin{tabular}{lc}
\hline \hline Sector & Representation \\
\hline$a a$ & $U\left(N_{a} / 2\right)$ vector multiplet and 3 adjoint chiral \\
multiplets \\
$a b+b a$ & $\mathcal{M}\left(\frac{N_{a}}{2}, \frac{N_{b}}{2}\right)=I_{a b}=2^{-k} \prod_{i=1}^{3}\left(n_{a}^{i} l_{b}^{i}-n_{b}^{i} l_{a}^{i}\right)$ \\
$a b^{\prime}+b^{\prime} a$ & $\mathcal{M}\left(\frac{N_{a}}{2}, \frac{N_{b}}{2}\right)=I_{a b^{\prime}}=-2^{-k} \prod_{i=1}^{3}\left(n_{a}^{i} l_{b}^{i}+n_{b}^{i} l_{a}^{i}\right)$ \\
$a a^{\prime}+a^{\prime} a$ & $\mathcal{M}\left(a_{S}\right)=\frac{1}{2}\left(I_{a a^{\prime}}-\frac{1}{2} I_{a O 6}\right) ;$ \\
& $\mathcal{M}\left(a_{A}\right)=\frac{1}{2}\left(I_{a a^{\prime}}+\frac{1}{2} I_{a O 6}\right)$ \\
\hline \hline
\end{tabular}

the internal space, resulting in a chiral fermion in the bifundamental representation localized at the intersection between different stacks. The multiplicity of such fermions is then given by the number of times the 3-cycles intersect. Due to orientifolding, for every stack of D6-branes we must also introduce its orientifold images. Thus, the D6-branes may also have intersections with the images of other stacks, also resulting in fermions in bifundamental representations. Each stack may also intersect its own images, resulting in chiral fermions in the symmetric and antisymmetric representations. The different types of representations that may be obtained for each type of intersection and their multiplicities are shown in Table I. In addition, there are constraints that must be satisfied for the consistency of the model, namely the requirement for Ramond-Ramond tadpole cancellation as well as to have a spectrum with $\mathcal{N}=1$ supersymmetry.

Intersecting D-brane configurations provide promising setups which may accommodate semirealistic features of low-energy physics. Given this, it is an interesting question to see how far one can get from a particular string compactification to reproducing the finer details of the Standard Model as a low-energy effective field theory. There have been many consistent models studied, but only a small number have the proper structures to produce an acceptable phenomenology. A good candidate for a realistic model which may possess the proper structures was discussed in $[5,6]$ in Type IIA theory on the $\mathbf{T}^{\mathbf{6}} /\left(\mathbb{Z}_{2} \times \mathbb{Z}_{2}\right)$ orientifold. We present the D6-brane configurations and intersection numbers of this model in Table II, and the resulting spectrum which is essentially that of a three-family Pati-Salam in Table III [6,7]. We put the $a^{\prime}, b$, and $c$ stacks of D6-branes on the top of each other on the third two torus, and as a result there are additional vectorlike particles from $N=2$ subsectors.

The anomalies from three global $U(1) \mathrm{s}$ of $U(4)_{C}$, $U(2)_{L}$, and $U(2)_{R}$ are canceled by the Green-Schwarz mechanism, and the gauge fields of these $U(1) \mathrm{s}$ obtain masses via the linear $B \wedge F$ couplings. Thus, the effective gauge symmetry is $S U(4)_{C} \times S U(2)_{L} \times S U(2)_{R}$. In order

TABLE II. D6-brane configurations and intersection numbers for the model on Type IIA $\mathbf{T}^{6} / \mathbb{Z}_{2} \times \mathbb{Z}_{2}$ orientifold. The complete gauge symmetry is $\left[U(4)_{C} \times U(2)_{L} \times U(2)_{R}\right]_{\text {observable }} \times\left[U S p(2)^{4}\right]_{\text {hidden }}$, the SM fermions and Higgs fields arise from the intersections on the first two-torus, and the complex structure parameters are $2 \chi_{1}=6 \chi_{2}=3 \chi_{3}=6$.

\begin{tabular}{|c|c|c|c|c|c|c|c|c|c|c|c|c|}
\hline & \multicolumn{12}{|c|}{$U(4)_{C} \times U(2)_{L} \times U(2)_{R} \times U S p(2)^{4}$} \\
\hline & $N$ & $\left(n^{1}, l^{1}\right) \times\left(n^{2}, l^{2}\right) \times\left(n^{3}, l^{3}\right)$ & $n_{S}$ & $n_{A}$ & $b$ & $b^{\prime}$ & $c$ & $c^{\prime}$ & 1 & 2 & 3 & 4 \\
\hline$a$ & 8 & $(0,-1) \times(1,1) \times(1,1)$ & 0 & 0 & 3 & 0 & -3 & 0 & 1 & -1 & 0 & 0 \\
\hline$b$ & 4 & $(3,1) \times(1,0) \times(1,-1)$ & 2 & -2 & $\ldots$ & $\ldots$ & 0 & 0 & 0 & 1 & 0 & -3 \\
\hline$c$ & 4 & $(3,-1) \times(0,1) \times(1,-1)$ & -2 & 2 & $\cdots$ & $\cdots$ & $\cdots$ & $\cdots$ & -1 & 0 & 3 & 0 \\
\hline 1 & 2 & $(1,0) \times(1,0) \times(2,0)$ & \multirow{4}{*}{\multicolumn{10}{|c|}{$\begin{array}{r}\chi_{1}=3, \chi_{2}=1, \chi_{3}= \\
\beta_{1}^{g}=-3, \beta_{2}^{g}=-3 \\
\beta_{3}^{g}=-3, \beta_{4}^{g}=-3\end{array}$}} \\
\hline 2 & 2 & $(1,0) \times(0,-1) \times(0,2)$ & & & & & & & & & & \\
\hline 3 & 2 & $(0,-1) \times(1,0) \times(0,2)$ & & & & & & & & & & \\
\hline 4 & 2 & $(0,-1) \times(0,1) \times(2,0)$ & & & & & & & & & & \\
\hline
\end{tabular}


TABLE III. The chiral and vectorlike superfields and their quantum numbers under the gauge symmetry $S U(4)_{C} \times S U(2)_{L} \times$ $\underline{S U(2)_{R} \times U S p(2)_{1} \times U S p(2)_{2} \times U S p(2)_{3} \times U S p(2)_{4} .}$

\begin{tabular}{lccccc}
\hline \hline & Quantum number & $Q_{4}$ & $Q_{2 L}$ & $Q_{2 R}$ & Field \\
\hline$a b$ & $3 \times(4, \overline{2}, 1,1,1,1,1)$ & 1 & -1 & 0 & $F_{L}\left(Q_{L}, L_{L}\right)$ \\
$a c$ & $3 \times(\overline{4}, 1,2,1,1,1,1)$ & -1 & 0 & 1 & $F_{R}\left(Q_{R}, L_{R}\right)$ \\
$a 1$ & $1 \times(4,1,1,2,1,1,1)$ & 1 & 0 & 0 & $X_{a 1}$ \\
$a 2$ & $1 \times(\overline{4}, 1,1,1,2,1,1)$ & -1 & 0 & 0 & $X_{a 2}$ \\
$b 2$ & $1 \times(1,2,1,1,2,1,1)$ & 0 & 1 & 0 & $X_{b 2}$ \\
$b 4$ & $3 \times(1, \overline{2}, 1,1,1,1,2)$ & 0 & -1 & 0 & $X_{b 4}^{i}$ \\
$c 1$ & $1 \times(1,1, \overline{2}, 2,1,1,1)$ & 0 & 0 & -1 & $X_{c 1}^{i}$ \\
$c 3$ & $3 \times(1,1,2,1,1,2,1)$ & 0 & 0 & 1 & $X_{c 3}^{i}$ \\
$b_{S}$ & $2 \times(1,3,1,1,1,1,1)$ & 0 & 2 & 0 & $T_{L}^{i}$ \\
$b_{A}$ & $2 \times(1, \overline{1}, 1,1,1,1,1)$ & 0 & -2 & 0 & $S_{L}^{i}$ \\
$c_{S}$ & $2 \times(1,1, \overline{3}, 1,1,1,1)$ & 0 & 0 & -2 & $T_{R}^{i}$ \\
$c_{A}$ & $2 \times(1,1,1,1,1,1,1)$ & 0 & 0 & 2 & $S_{R}^{i}$ \\
$a b^{\prime}$ & $3 \times(4,2,1,1,1,1,1)$ & 1 & 1 & 0 & \\
& $3 \times(\overline{4}, \overline{2}, 1,1,1,1,1)$ & -1 & -1 & 0 & \\
$a c^{\prime}$ & $3 \times(4,1,2,1,1,1,1)$ & 1 & & 1 & $\Phi_{i}$ \\
& $3 \times(\overline{4}, 1, \overline{2}, 1,1,1,1)$ & -1 & 0 & -1 & $\bar{\Phi}_{i}$ \\
$b c$ & $6 \times(1,2, \overline{2}, 1,1,1,1)$ & 0 & 1 & -1 & $H_{u}^{i}, H_{d}^{i}$ \\
& $6 \times(1, \overline{2}, 2,1,1,1,1)$ & 0 & -1 & 1 & \\
\hline \hline
\end{tabular}

to break the gauge symmetry, on the first torus, we split the $a$ stack of D6-branes into $a_{1}$ and $a_{2}$ stacks with 6 and 2 D6-branes, respectively, and split the $c$ stack of D6-branes into $c_{1}$ and $c_{2}$ stacks with two D6-branes for each one. In this way, the gauge symmetry is further broken to $S U(3)_{C} \times S U(2)_{L} \times U(1)_{I_{3 R}} \times U(1)_{B-L}$. Moreover, the $U(1)_{I_{3 R}} \times U(1)_{B-L}$ gauge symmetry may be broken to $U(1)_{Y}$ by giving vacuum expectation values (VEVs) to the vectorlike particles with the quantum numbers $(\mathbf{1}, \mathbf{1}, \mathbf{1} / \mathbf{2},-\mathbf{1})$ and $(\mathbf{1}, \mathbf{1},-\mathbf{1} / \mathbf{2}, \mathbf{1})$ under the $S U(3)_{C} \times$ $S U(2)_{L} \times U(1)_{I_{3 R}} \times U(1)_{B-L}$ gauge symmetry from $a_{2} c_{1}^{\prime}$ intersections [7].

Since the gauge couplings in the minimal supersymmetric standard model (MSSM) are unified at the GUT scale $\sim 2.4 \times 10^{16} \mathrm{GeV}$, the additional exotic particles present in the model must necessarily become superheavy. To accomplish this it is first assumed that the $\operatorname{USp}(2)_{1}$ and $U S p(2)_{2}$ stacks of D6-branes lie on top of each other on the first torus, so we have two pairs of vectorlike particles $X_{12}^{i}$ with $U S p(2)_{1} \times U S p(2)_{2}$ quantum numbers $(2,2)$. These particles can break $U S p(2)_{1} \times U S p(2)_{2}$ down to the diagonal $\operatorname{USp}(2)_{D 12}$ near the string scale by obtaining VEVs, and then states arising from intersections $a 1$ and $a 2$ may obtain vectorlike masses close to the string scale from superpotential terms of the form

$$
W \subset X_{a 1} X_{a 2} X_{12}^{i},
$$

where we neglect the couplings of order one. Moreover, we assume that the $T_{R}^{i}$ and $S_{R}^{i}$ obtain VEVs near the string scale, and their VEVs satisfy the D-flatness of $U(1)_{R}$. We also assume that there exist various suitable highdimensional operators in the effective theory, and thus the adjoint chiral superfields may obtain GUT-scale masses via these operators. With $T_{R}^{i}$ and $S_{R}^{i}$, we can give GUT-scale masses to the particles from the intersections $c 1, c 3$, and $c_{S}$ via the superpotential:

$$
W \subset S_{R}^{i} X_{c 1} X_{c 1}+T_{R}^{i} X_{c 3}^{j} X_{c 3}^{k}+\frac{1}{M_{\mathrm{Pl}}} S_{R}^{i} S_{R}^{j} T_{R}^{k} T_{R}^{l} .
$$

The beta function for $U S p(2)_{D 12}$ is -4 and the gauge coupling for $U S p(2)_{D 12}$ will become strongly coupled around $5 \times 10^{12} \mathrm{GeV}$, and then we can give $5 \times 10^{12} \mathrm{GeV}$ scale VEVs to $S_{L}^{i}$ and preserve the D-flatness of $U(1)_{L}$. The remaining states may also obtain intermediate scale masses via the operators

$$
W \subset X_{b 2} X_{b 2} S_{l}^{i}+\frac{X_{b 2} X_{b 2}}{M_{X}} X_{b 4} X_{b 4}
$$

To have one pair of light Higgs doublets, it is necessary to fine-tune the mixing parameters of the Higgs doublets. In particular, the $\mu$ term and the right-handed neutrino masses may be generated via the following high-dimensional operators

$$
W \supset \frac{y_{\mu}^{i j k l}}{M_{\mathrm{St}}} S_{L}^{i} S_{R}^{j} H_{u}^{k} H_{d}^{l}+\frac{y_{N i j}^{m n k l}}{M_{\mathrm{St}}^{3}} T_{R}^{m} T_{R}^{n} \Phi_{i} \Phi_{j} F_{R}^{k} F_{R}^{l},
$$

where $y_{\mu}^{i j k l}$ and $y_{N i j}^{m n k l}$ are Yukawa couplings, and $M_{\mathrm{St}}$ is the string scale. Thus, the $\mu$ term is $\mathrm{TeV}$ scale and the righthanded neutrino masses can be in the range $10^{10-14} \mathrm{GeV}$ for $y_{\mu}^{i j k l} \sim 1$ and $y_{N i j}^{m n k l} \sim 10^{(-7)-(-3)}$.

In intersecting D-brane models, the gauge groups arise from different stacks of branes, and so they will not generally have the same volume in the compactified space. Thus, the gauge couplings are not automatically unified, in contrast to heterotic models. However, for this model, the gauge couplings turn out to be unified at the string scale [6].

\section{NEUTRINO MASSES AND MIXING ANGLES}

In recent years, precision measurements of the neutrino mixing angles as well as the differences of the masses squared between neutrinos have been made by several experiments. Initially, the best estimate of the difference in the squares of the masses of mass eigenstates 1 and 2 was published by KamLAND in 2005. However, more recent studies have indicated that the $\Delta m_{21}^{2}$ values preferred by KamLAND are somewhat higher than results from solar experiments [8]. With that said the latest global analysis of the data produces $\Delta m_{21}^{2}=0.0000739_{-0.0000020}^{+0.0000021} \mathrm{eV}^{2}$ 
[8-11]. In addition, the MINOS experiment measured oscillations from an intense muon neutrino beam, determining the difference in the squares of the masses between neutrino mass eigenstates 2 and 3. Current measurements indicate $\Delta m_{32}^{2}=0.002523_{-0.000030}^{+0.000032} \mathrm{eV}^{2}[8,10,11]$, consistent with previous results from Super-Kamiokande [12]. In addition, recent analysis of cosmological results constrains the sum of the three neutrino masses to be $\lesssim 0.12 \mathrm{eV}$ [13], while additional analysis of combined datasets results in $0.15 \mathrm{eV}$ [14] and $0.19 \mathrm{eV}$ [15] for the upper limit. Older analyses set the upper limit slightly higher at $0.3 \mathrm{eV}$ [16-18].

The Pontecorvo-Maki-Nakagawa-Sakata (PMNS) lepton mixing matrix may be parametrized as

$$
U_{\mathrm{PMNS}}=\left(\begin{array}{ccc}
c_{12} c_{13} & s_{12} c_{13} & s_{13} e^{-i \delta_{C P}} \\
-s_{12} c_{23}-c_{12} s_{23} s_{13} e^{i \delta_{C P}} & c_{12} c_{23}-s_{12} s_{23} s_{13} e^{i \delta_{C P}} & s_{23} c_{13} \\
s_{12} s_{23}-c_{12} c_{23} s_{13} e^{i \delta_{C P}} & -c_{12} s_{23}-s_{12} c_{23} s_{13} e^{i \delta_{C P}} & c_{23} c_{13}
\end{array}\right),
$$

where $s_{i j}$ and $c_{i j}$ denote $\sin \theta_{i j}$ and $\cos \theta_{i j}$, respectively, while $\delta_{C P}$ is a $C P$-violating phase.

The current best-fit values for the mixing angles from direct and indirect experiments are, using normal ordering [8,10,11],

$$
\theta_{12}=33.82_{-0.76^{\circ}}^{\circ+0.78^{\circ}}, \quad \theta_{23}=48.3_{-1.9^{\circ}}^{\circ+1 . \circ^{\circ}}, \quad \theta_{13}=8.61_{-0.13^{\circ}}^{\circ+0.13^{\circ}}, \quad \delta_{C P}=222_{-28^{\circ}}^{\circ+38^{\circ}}
$$

Using these values, the $3 \sigma$ ranges on the PMNS matrix [11] are given by

$$
|V|_{\text {PMNS }}^{3 \sigma}=\left(\begin{array}{lll}
0.797 \rightarrow 0.842 & 0.518 \rightarrow 0.585 & 0.143 \rightarrow 0.156 \\
0.244 \rightarrow 0.496 & 0.467 \rightarrow 0.678 & 0.646 \rightarrow 0.772 \\
0.287 \rightarrow 0.525 & 0.488 \rightarrow 0.693 & 0.618 \rightarrow 0.749
\end{array}\right)
$$

One of the most studied patterns of neutrino mixing angles is the so-called tribimaximal mixing of the form

$$
U_{T B} \sim\left(\begin{array}{ccc}
\sqrt{\frac{2}{3}} & \sqrt{\frac{1}{3}} & 0 \\
-\sqrt{\frac{1}{6}} & \sqrt{\frac{1}{3}} & -\sqrt{\frac{1}{2}} \\
-\sqrt{\frac{1}{6}} & \sqrt{\frac{1}{3}} & \sqrt{\frac{1}{2}}
\end{array}\right)
$$

which was consistent with early data. However, the measurement of a nonzero $\theta_{13}$ by Data Bay [19] and Double Chooz [20], and confirmed by RENO [21], has now ruled out these mixing patterns. However, tribimaximal mixing may still be viewed as a zeroth-order approximation to more general forms of the PMNS matrix which are also consistent with the current data. Thus, it is still of great importance to understand the origin of tribimaximal mixing.

It has been shown that mass matrices leading to tribimaximal and near-tribimaximal mixing may be generated by imposing a flavor symmetry such as $\mathbf{A 4}$ [22] or $\boldsymbol{\Delta}(27)$ [23]. Specifically, a mass matrix of the form

$$
\mathcal{M}_{\nu} \sim\left(\begin{array}{ccc}
Y & X & X \\
X & y & x \\
X & x & y
\end{array}\right)
$$

obtained by imposing an A4 flavor symmetry leads to tribimaximal mixing, while mass matrices of the form

$$
\mathcal{M}_{\nu} \sim\left(\begin{array}{lll}
f_{1} v_{1} & f_{2} v_{3} & f_{2} v_{2} \\
f_{2} v_{3} & f_{1} v_{2} & f_{2} v_{1} \\
f_{2} v_{2} & f_{2} v_{1} & f_{1} v_{3}
\end{array}\right)
$$

obtained by imposing an $\boldsymbol{\Delta}(\mathbf{2 7})$ flavor symmetry, may lead to near-tribimaximal mixing. It is shown in the next section that the Yukawa matrices in a particular intersecting D-brane model may naturally be of this form.

In order to naturally explain the smallness of the neutrino masses in comparison to the other fermion masses, a seesaw mechanism is usually invoked. In the canonical or Type I seesaw, the Majorana mass matrix for left-handed neutrinos is given by

$$
M_{\nu}^{M}=-M_{\nu}^{D} M_{R}^{-1}\left(M_{\nu}^{D}\right)^{T}
$$

where $M_{\nu}^{D}$ is the Dirac mass matrix for neutrinos and $M_{R}$ is the right-handed neutrino mass matrix.

\section{FERMION MASS MATRICES IN A REALISTIC STRING MODEL}

In the past two decades, a promising approach to string model building has emerged involving compactifications 
with D-branes on orientifolds (for reviews, see [24-27]). In such models chiral fermions-an intrinsic feature of the SM-arise from configurations with D-branes located at transversal orbifold/conifold singularities [28] and strings stretching between D-branes intersecting at angles $[29,30]$ (or, in its T-dual picture, with magnetized D-branes [31-33]).

Within the framework of D-brane modeling it was demonstrated that the Yukawa matrices $Y_{a b c} \sim$ $\exp \left(-A_{a b c}\right)$ arise from world-sheet areas $A_{a b c}$ spanning $\mathrm{D}$ branes (labeled by $a, b, c$ ) supporting fermions and Higgses at their intersections [30,34]. This pattern naturally encodes the hierarchy of Yukawa couplings. In addition, due to the internal geometry of these compactifications as well as due to stringy selection rules present in such models, discrete flavor symmetries may arise. In particular, it has been shown that discrete symmetries such as $D_{4}$ and $\Delta(27)$ may naturally arise in such models [35].

However, for most string constructions, the Yukawa matrices are of rank one. In the case of D-brane models built on toroidal orientifolds, this result can be traced to the fact that not all of the intersections at which the SM fermions are localized occur on the same torus. To date only one threegeneration model is known in which this problem has been overcome [5,6], and for which one can obtain mass matrices for quarks and leptons that may reproduce the experimentally observed values. Additionally, this model exhibits automatic gauge coupling unification at the string scale, and all extra matter can be decoupled.

In this model, the Yukawa couplings for the quarks and leptons are all allowed and are given by the superpotential

$$
\begin{aligned}
W_{Y}= & Y_{i j k}^{U} Q_{L}^{i} U_{R}^{j} H_{U}^{k}+Y_{i j k}^{D} Q_{L}^{i} D_{R}^{j} H_{D}^{k}+Y_{i j k}^{\nu} L^{i} N^{j} H_{U}^{k} \\
& +Y_{i j k}^{L} L^{i} E^{j} H_{D}^{k},
\end{aligned}
$$

where $Q_{L}^{i} i$ and $L^{i}$ are the left-handed quark and lepton fields, respectively, while $U_{R}^{j}, D_{R}^{j}, N^{j}$, and $E^{j}$ are the righthanded up quarks, down quarks, neutrinos, and charged leptons, respectively, and $H_{U}^{k}$ and $H_{D}^{k}$ are the up-type and down-type Higgs fields, with

$$
i \in\{0,1,2\}, \quad j \in\{0,1,2\}, \quad k \in\{0,1,2,3,4,5\} .
$$

In addition, the $\mu$ term and right-handed neutrino masses which may be generated via the higher-dimensional are shown in Eq. (4).

A complete form for the Yukawa couplings $y_{i j}^{f}$ for D6-branes wrapping on a full compact space $T^{2} \times T^{2} \times T^{2}$ can be expressed as $[34,36]$

$$
Y_{\{i j k\}}=h_{q u} \sigma_{a b c} \prod_{r=1}^{3} \vartheta\left[\begin{array}{l}
\delta^{(r)} \\
\phi^{(r)}
\end{array}\right]\left(\kappa^{(r)}\right)
$$

where

$$
\vartheta\left[\begin{array}{l}
\delta^{(r)} \\
\phi^{(r)}
\end{array}\right]\left(\kappa^{(r)}\right)=\sum_{l \in \mathbf{Z}} e^{\pi i\left(\delta^{(r)}+l\right)^{2} \kappa^{(r)}} e^{2 \pi i\left(\delta^{(r)}+l\right) \phi^{(r)}},
$$

with $r=1,2,3$ denoting the three two-tori. For the present model, we focus on the first torus $(r=1)$ as the other twotori only produce an overall constant.

The parameter $\delta^{(1)}$ is a function of $i, j$, and $k$ and is given by

$$
\delta^{(1)}=\frac{i}{3}-\frac{j}{3}-\frac{k}{6}+\frac{s}{3}+\epsilon_{(a, b)}^{(1)} .
$$

By choosing a different linear function for $s^{(1)}$, some independent modes with nonzero eigenvalues are possible. Specifically, we will consider the case $s^{(1)}=-i$ so that

$$
\delta^{(1)}=-\frac{j}{3}-\frac{k}{6}+\epsilon_{(a, b)}^{(1)} .
$$

Here, the parameter $\epsilon(1)_{a, b l}$ is an overall shift parameter which depends upon the positions of each stack of D-branes in the internal space. Thus for a quark or lepton field localized at the intersection between stacks $a$ and $b$, the shift parameter $\epsilon_{(a, b)}$ depends on the positions of stacks $a$ and $b$ in the internal space. In order to have a consistent solution, the shift parameters for each type of fermion must satisfy the constraint,

$$
\epsilon_{u}^{(r)}+\epsilon_{l}^{(r)}=\epsilon_{d}^{(r)}+\epsilon_{\nu}^{(r)} .
$$

Likewise, the parameter $\kappa^{(1)}=\frac{6 J^{(1)}}{\alpha^{\prime}}$ is a scale factor related to the Kähler modulus $J^{(1)}$, while $\phi^{(r)}$ is an effective Wilson line for each torus. See [2] for the full definition of these parameters.

In addition, there is a selection rule,

$$
i+j+k=0 \bmod 3,
$$

which determines which Higgs fields couple to the different quark and lepton fields. The Yukawa matrices in this model are then of the form

$\mathcal{M} \sim\left(\begin{array}{lll}A v_{1} & B v_{3} & C v_{5} \\ C v_{3} & A v_{5} & B v_{1} \\ B v_{5} & C v_{1} & A v_{3}\end{array}\right)+\left(\begin{array}{ccc}E v_{4} & F v_{6} & D v_{2} \\ D v_{6} & E v_{2} & F v_{4} \\ F v_{2} & D v_{4} & E v_{6}\end{array}\right)$,

where $v_{k}=\left\langle H_{k+1}\right\rangle$ and the Yukawa couplings $A, B, C, D$, $E$, and $F$ are given by 


$$
\begin{array}{ll}
A \equiv \vartheta\left[\begin{array}{c}
\epsilon^{(1)} \\
\phi^{(1)}
\end{array}\right]\left(\frac{6 J^{(1)}}{\alpha^{\prime}}\right), & B \equiv \vartheta\left[\begin{array}{c}
\epsilon^{(1)}+\frac{1}{3} \\
\phi^{(1)}
\end{array}\right]\left(\frac{6 J^{(1)}}{\alpha^{\prime}}\right), \\
C \equiv \vartheta\left[\begin{array}{c}
\epsilon^{(1)}-\frac{1}{3} \\
\phi^{(1)}
\end{array}\right]\left(\frac{6 J^{(1)}}{\alpha^{\prime}}\right), & D \equiv \vartheta\left[\begin{array}{c}
\epsilon^{(1)}+\frac{1}{6} \\
\phi^{(1)}
\end{array}\right]\left(\frac{6 J^{(1)}}{\alpha^{\prime}}\right), \\
E \equiv \vartheta\left[\begin{array}{c}
\epsilon^{(1)}+\frac{1}{2} \\
\phi^{(1)}
\end{array}\right]\left(\frac{6 J^{(1)}}{\alpha^{\prime}}\right), & F \equiv \vartheta\left[\begin{array}{c}
\epsilon^{(1)}-\frac{1}{6} \\
\phi^{(1)}
\end{array}\right]\left(\frac{6 J^{(1)}}{\alpha^{\prime}}\right) .
\end{array}
$$

These Yukawa matrices are of rank 3, such that it is possible to have three different mass eigenvalues as well as nontrivial mixing between each of the different generations. In the MSSM, the up-type quarks and neutrinos receive mass from the isospin up Higgs field $H_{U}$, while the downtype quarks and charged leptons receive mass from the isospin down Higgs field $H_{D}$. In this model, there are actually six different Higgs fields in each sector. We may fine-tune the Higgsino bilinear $\mu$ term given in Eq. (4) such that there are only two massless eigenstates given by

$$
H_{u, d}=\sum_{i} \frac{v_{u, d \mid}^{i} H_{u, d}^{i}}{\sqrt{\sum\left(v_{u, d}^{i}\right)^{2}}}
$$

where $v_{u, d}^{i}=\left\langle H_{u, d}^{i}\right\rangle$. In fitting the mass matrices, we treat the Higgs VEVs as free parameters. However, these parameters may ultimately be calculated in the model. By choosing the shift parameter to be $\epsilon=0$ for quarks and $\epsilon=1 / 2$ for leptons (or vice versa), the mass matrices are of the same form as those obtained by imposing a $\Delta(27)$ discrete flavor symmetry given in Eq. (10), since for these values of the shift parameters, $B=C$ and $D=F$. In addition with this choice, the up- and down-type quarks predominantly receive masses mainly via the odd-numbered Higgs VEVs $v_{\text {odd }}^{U, D}$ while the neutrinos and charged leptons obtain mass predominately via the even-numbered
Higgs VEVs $v_{\text {even }}^{U, D}$, or vice versa. However, it should be emphasized that the fermions in each sector couple to all of the Higgs fields in each sector. Thus, the mass matrix for the up-type quarks is not independent from the neutrino mass matrix, and likewise for the down-type quarks and the charged leptons.

Our strategy then is to choose the Higgs VEVs and the Kähler parameter $\kappa$ in order to obtain mass matrices for the quarks and leptons that give the correct mass eigenvalues as well as the correct CKM matrix. In particular, we fit them for $\tan \beta=50$ as shown in [2]. This may be accomplished by choosing the following values for the Higgs VEVs:

$$
\begin{aligned}
v_{u}^{1} & =0.0000142, & v_{d}^{1} & =0.0028224, \\
v_{u}^{2} & =0.00002408185, & v_{d}^{2} & =0.045, \\
v_{u}^{3} & =1.0, & v_{d}^{3} & =1.0, \\
v_{u}^{4} & =0.000000345, & v_{d}^{4} & =0.0010105, \\
v_{u}^{5} & =0.00404, & v_{d}^{5} & =0.0266, \\
v_{u}^{6} & =0.005960855, & v_{d}^{6} & =1.0 .
\end{aligned}
$$

Nearly the correct CKM matrix is then obtained by choosing $\kappa=58.7$. Note that the Higgs VEVs $v_{u}^{\text {even }}$ have been chosen so that the neutrino mass matrix will be neartribimaximal, i.e., in the form given in Eq. (9). The values for VEVs $v^{\text {even }}$ required to do this are then determined by the off-diagonal elements of the up-type quark mass matrix when those Yukawa couplings are evaluated at $\epsilon=0.5$. Thus, the up-type quark mass matrix and the Dirac neutrino mass matrix are not independent of each other. Once the uptype quark matrix is determined, the requirement that the neutrino matrix result in tribimaximal mixing completely fixes it. For example, after fixing the odd-numbered uptype Higgs VEVs, the neutrino mass matrix with $\epsilon_{\nu}=0.5$ is given by

$$
M_{\nu}=m_{t}\left(\begin{array}{ccc}
E v_{u}^{4} & 0.005960855+F v_{u}^{6} & 0.0002408185+D v_{u}^{2} \\
0.005960855+D v_{u}^{6} & 0.00005+E v_{u}^{2} & 0.00000008464414+F v_{u}^{4} \\
0.0002408185+F v_{u}^{2} & 0.00000008464414+D v_{u}^{4} & E v_{u}^{6}
\end{array}\right) .
$$

Then $v_{u}^{2}, v_{u}^{4}, v_{u}^{6}$ may be chosen so that the neutrino mass matrix is of the form given in Eq. (9) which results in tribimaximal mixing.

We set all $C P$ phases to zero by setting the Wilson lines, $\phi$ which are input into the Jacobi Theta functions equal to zero. However, note that they may also be included in the fit so that the Dirac $C P$ violating phase, which appears in the neutrino mixing matrix, may be determined once the $C P$ violating phases in the quark sector are fit. With these parameters, the mass matrices for the up- and down-type quarks are given by

$$
M_{u}=m_{t}\left(\begin{array}{ccc}
0.0000142 & 0.00003553304 & 0.0000001436 \\
0.00003553304 & 0.00404 & 0.000000002055 \\
0.0000001436 & 0.000000002055 & 1
\end{array}\right)
$$




$$
M_{d}=m_{b}\left(\begin{array}{ccc}
0.0028224 & 0.005960856 & 0.0002682385 \\
0.005960856 & 0.0266 & 0.000006023448 \\
0.0002682385 & 0.000006023448 & 1
\end{array}\right) \text {, }
$$

whose eigenvalues have the correct quark mass hierarchies and nearly the correct CKM matrix is obtained. Similarly, the mass matrices for the neutrinos and charged leptons are given by

$$
\begin{gathered}
M_{\nu}=m_{t}\left(\begin{array}{ccc}
0.000000345 & 0.005960855 & 0.00002408185 \\
0.005960855 & 0.00002408185 & 0.00000008464414 \\
0.00002408185 & 0.00000008464414 & 0.005960855
\end{array}\right), \\
M_{l}=m_{b}\left(\begin{array}{ccc}
0.0010105 & 0.005960856 & 0.0001585588 \\
0.005960856 & 0.045 & 0.00001682392 \\
0.0001585588 & 0.00001682392 & 1
\end{array}\right) .
\end{gathered}
$$

The eigenvalues for the charged lepton mass matrix then have the correct mass hierarchy, while the Dirac neutrino mass matrix is near tribimaximal and the charged lepton mass matrix is near diagonal.

\section{RGE EVOLUTION TO THE ELECTROWEAK SCALE}

The Yukawa mass matrices obtained in the previous section are obtained at the string scale, which in the following we will take to be the same as the GUT scale, $M_{X}=2 \times 10^{16} \mathrm{GeV}$. In order to compare the model predictions with experimental results it is necessary to evolve these mass matrices down to the electroweak scale via the RGE, as well as apply a seesaw mechanism. In order to do this we use the REAP 11.4 Mathematica package [4].

The Yukawa couplings are given by

$$
y_{t}=\frac{\sqrt{2} m_{t}}{v \cdot \sin \beta}, \quad y_{b}=\frac{\sqrt{2} m_{b}}{v \cdot \cos \beta},
$$

where $m_{t}=129 \mathrm{GeV}$ and $m_{b}=1.00 \mathrm{GeV}$ at $M_{\mathrm{GUT}}$. For $\tan \beta=50$, we then have that $y_{t}=0.742$ and $y_{b}=0.286$. The Yukawa matrices are then given by

$$
\begin{gathered}
Y_{u}=y_{t}\left(\begin{array}{ccc}
0.0000142 & 0.00003553304 & 0.0000001436 \\
0.00003553304 & 0.00404 & 0.000000002055 \\
0.0000001436 & 0.000000002055 & 1
\end{array}\right), \\
Y_{d}=y_{b}\left(\begin{array}{ccc}
0.0028224 & 0.005960856 & 0.0002682385 \\
0.005960856 & 0.0266 & 0.000006023448 \\
0.0002682385 & 0.000006023448 & 1
\end{array}\right),
\end{gathered}
$$

and

$$
\begin{gathered}
Y_{\nu}=y_{t}\left(\begin{array}{ccc}
0.000000345 & 0.005960855 & 0.00002408185 \\
0.005960855 & 0.00002408185 & 0.00000008464414 \\
0.00002408185 & 0.00000008464414 & 0.005960855
\end{array}\right), \\
Y_{l}=y_{b}\left(\begin{array}{ccc}
0.0010105 & 0.005960856 & 0.0001585588 \\
0.005960856 & 0.045 & 0.00001682392 \\
0.0001585588 & 0.00001682392 & 1
\end{array}\right) .
\end{gathered}
$$




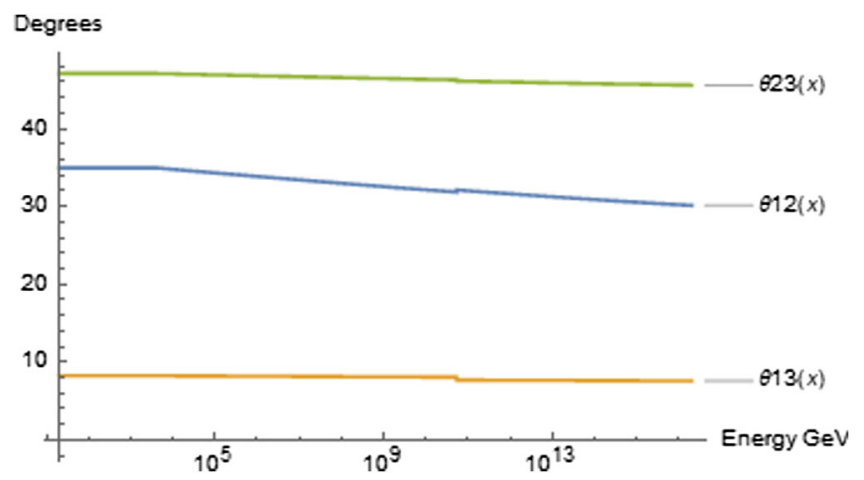

FIG. 1. Neutrino mixing angles as a function of the energy scale. Note that we have taken the supersymmetry decoupling scale to be 2-7 $\mathrm{TeV}$ in order to obtain the best agreement with data.

In addition to the Yukawa matrices for quarks and leptons, we must choose a right-handed neutrino mass matrix to be input into the seesaw mechanism, resulting in the mass matrix for left-handed neutrinos given by Eq. (11). In principle, this may be calculated in the model. However, the right-handed neutrino mass term in the superpotential arises from dimension-five operators as shown in Eq. (4), and thus it is very difficult to calculate. Therefore, for the present work we will choose the right-handed neutrino mass matrix such that the near-tribimaximal neutrino mixing originating in the Dirac neutrino mass matrix is preserved when running the RGEs from the string scale down to the GUT scale. A scan over a large number of random matrices produced 20000 different choices for the right-handed neutrino matrix. These right-handed neutrino matrices produced neutrino masses which give mass-squared differences within the experimentally observed ranges with a ratio of $\Delta m_{32}^{2} / \Delta m_{21}^{2}>30$. These right-handed neutrino matrices also produced neutrino masses whose sum is lower than the upper limit from cosmological data defined as

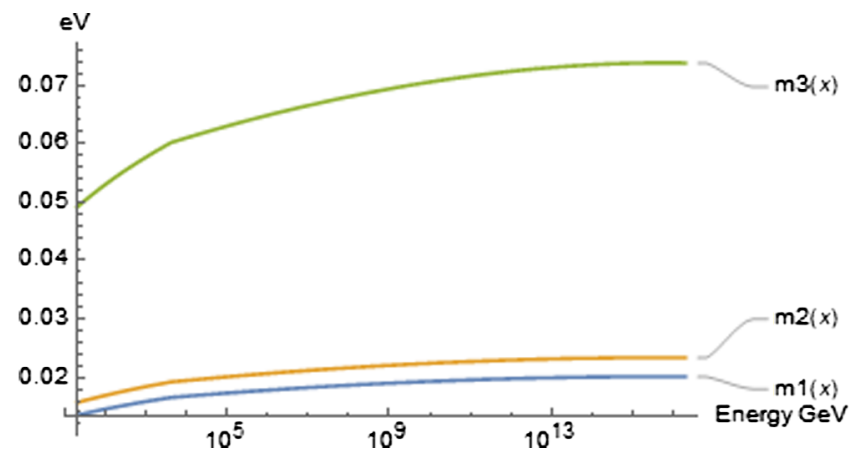

FIG. 2. Neutrino masses as a function of the energy scale. Note that we have taken the supersymmetry decoupling scale to be 2-7 TeV in order to obtain the best agreement with data.

$\sum_{i} m_{i} \lesssim 0.12 \mathrm{eV}$, and results in a PMNS matrix within the $3 \sigma$ ranges given in Eq. (7). The values for the right-handed neutrino matrix elements which satisfy these constraints are

$M_{R} \approx M_{r}\left(\begin{array}{ccc}-5.01 \pm 0.05 & -0.24 \pm 0.01 & 0.25 \pm 0.01 \\ -0.24 \pm 0.01 & -3.12 \pm 0.04 & 1.69 \pm 0.07 \\ 0.25 \pm 0.01 & 1.69 \pm 0.07 & -3.22 \pm 0.07\end{array}\right)$

where $M_{r}=10^{10} \mathrm{GeV} .{ }^{1}$ Inserting these values into the REAP package, we then find that the neutrino mixing angles at the electroweak scale are given by

$\theta_{12}=33.8^{\circ} \pm 1.2^{\circ}, \quad \theta_{23}=46.9^{\circ} \pm 0.9^{\circ}$,

$\theta_{13}=8.56^{\circ} \pm 0.20^{\circ}$,

where we give an uncertainty based upon how these results change when the right-handed neutrino mass parameters are varied. Using these values for the mixing angles, the PMNS lepton mixing matrix is then given by

$$
\left|V_{\mathrm{PMNS}}\right| \approx\left(\begin{array}{ccc}
0.822 \pm 0.01 & 0.549 \pm 0.01 & 0.149 \pm 0.003 \\
0.470 \pm 0.012 & 0.5081 \pm 0.016 & 0.721 \pm 0.010 \\
0.321 \pm 0.017 & 0.663 \pm 9.011 & 0.6676 \pm 0.011
\end{array}\right)
$$

which is in excellent agreement with the $3 \sigma$ limits given in Eq. (7). A plot of the neutrino mixing angles as a function of the energy scale is shown in Fig. 1, while the running of the neutrino masses is shown in Fig. 2.

In addition, we find that the neutrino masses at the electroweak scale are given by

\footnotetext{
${ }^{1}$ It may also be possible to employ an inverse seesaw mechanism using lower dimensional operators which are easier to calculate.
}

$$
\begin{aligned}
& m_{1}=0.0150 \pm 0.0002 \mathrm{eV}, \\
& m_{2}=0.0173 \pm 0.0002 \mathrm{eV}, \\
& m_{3}=0.053 \pm 0.002 \mathrm{eV},
\end{aligned}
$$

with

$$
\sum_{i} m_{i}=0.085 \pm 0.002 \mathrm{eV}
$$

consistent with cosmological constraints. Finally, we find that 


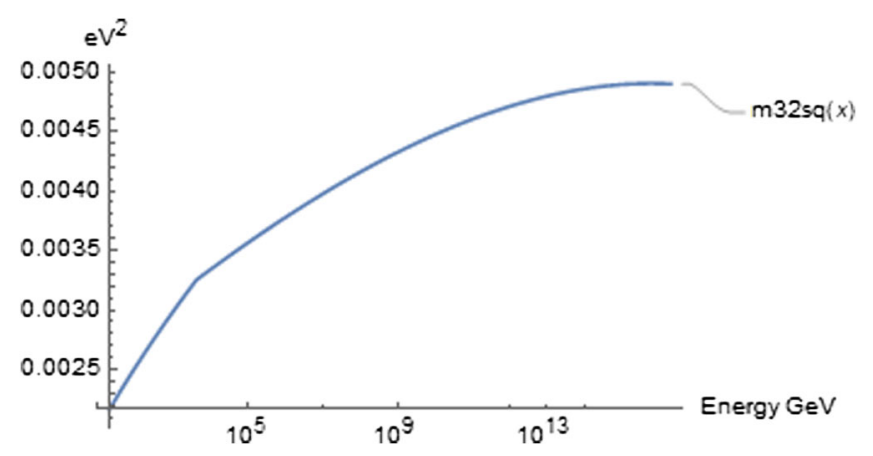

FIG. 3. Mass difference $\Delta m_{32}^{2}$ as a function of the energy scale. Note that we have taken the supersymmetry decoupling scale to be $2-7 \mathrm{TeV}$ in order to obtain the best agreement with data.

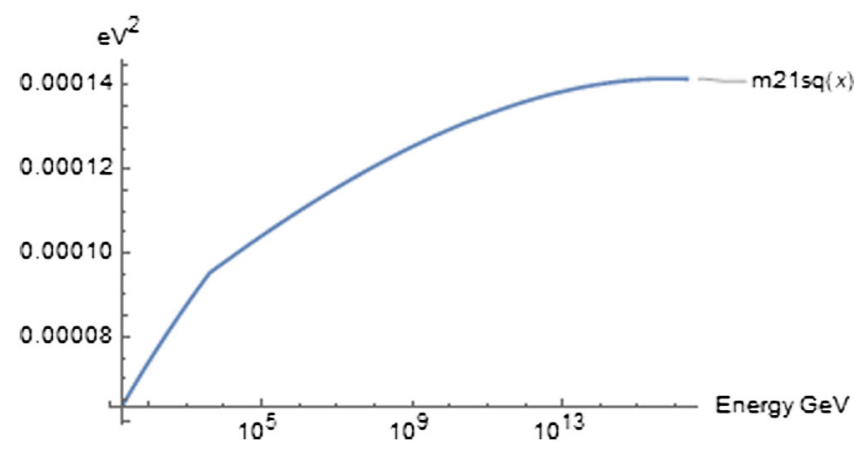

FIG. 4. Mass difference $\Delta m_{21}^{2}$ as a function of the energy scale. Note that we have taken the supersymmetry decoupling scale to be $2-7 \mathrm{TeV}$ in order to obtain the best agreement with data.

$$
\begin{aligned}
& \Delta m_{32}^{2}=0.0025 \pm 0.0002 \mathrm{eV}^{2}, \\
& \Delta m_{21}^{2}=0.000075 \pm 0.000003 \mathrm{eV}^{2} .
\end{aligned}
$$

These values are consistent with current experimental observations of neutrino oscillations. Plots of $m_{32}^{2}$ and $m_{21}^{2}$ as functions of the energy scale are shown in Figs. 3 and 4. Note that we have taken the supersymmetry decoupling scale to be $2-7 \mathrm{TeV}$ in order to obtain the best agreement with data. Thus, a change of slope in the RGE plots may be seen beginning at this scale.

\section{CONCLUSION}

We have performed an RGE analysis of the neutrino masses and mixing angles in a realistic Pati-Salam model constructed from intersecting D6 branes on a $T^{6} /\left(\mathbb{Z}_{2} \times \mathbb{Z}_{2}\right)$ orientifold. In previous work it had been shown that it is possible to fit the quark and lepton Yukawa matrices in the model such that the correct masses are obtained for the quarks and charged leptons as well as the nearly correct CKM quark mixing matrix. In addition, a Dirac neutrino mass matrix which is nearly tribimaximal was naturally obtained. A suitable right-handed neutrino mass matrix was chosen and then inserted into a Type I seesaw mechanism along with the Dirac neutrino mass matrix. The neutrino mass parameters were then evolved from the GUT scale down to the electroweak scale using the REAP Mathematica package. We then obtained neutrino masses given by $m_{1}=0.015 \pm 0.0002 \mathrm{eV}, m_{2}=$ $0.0173 \pm 0.0002 \mathrm{eV}$, and $m_{3}=0.053 \pm 0.002 \mathrm{eV}$, while simultaneously obtaining electroweak scale mixing angles and neutrino mass-squared differences which are within current experimental limits.

In fitting the SM fermion masses and mixings we have made use of several free parameters. In particular, the free parameters are the 12 Higgs VEVs, the Kähler parameter $\kappa$, and the five independent parameters in the right-handed neutrino mass matrix. In addition there are four shift parameters which are fixed by the $\Delta(27)$ constraint on the mass matrices. In addition, there is the effective supersymmetry decoupling scale in the RGE analysis which we have fixed by requiring the neutrino masssquared differences to be consistent with current experimental data. Therefore, there is nominally a total of 18 free parameters in the analysis. However, since these parameters are not independent of each other, there is effectively fewer than 18. With these parameters we have fit 12 quark and lepton masses and six quark and lepton mixing angles for a total of 18 observable quantities. Therefore, there are no more free parameters than there are observable quantities. Thus, as the fit is highly constrained, the obtained values for the neutrino masses may be regarded as a bona fide prediction of the model.

In principle, it may be possible to determine all of the adjustable parameters within the model. For example, by calculating the Higgsino bilinear mass matrix given in Eq. (22) it may be possible to determine the values of the Higgs VEVs. In particular, they would correspond to the coefficients for the massless eigenstates corresponding to $H_{U}$ and $H_{D}$. Similarly, the right-handed neutrino mass matrix may in principle be calculated within the model. Then, it might be possible to calculate the observed masses and mixing angles for the quarks and leptons from first principles. Another possibility is that the observed $\mathrm{CP}$ violating phases appearing in the CKM matrix may be included in the fit, allowing the Dirac $C P$ phase appearing in the lepton mixing matrix to be predicted. We plan to explore these possibilities in future work.

\section{ACKNOWLEDGMENTS}

E. H. was supported by the grant Pathways to STEM Careers, funded by the HSI STEM program of the Department of Education (Award No. P031C160242). 
[1] M. Abbas and S. Khalil, Phys. Rev. D 91, 053003 (2015).

[2] V. E. Mayes, Nucl. Phys. B950, 114848 (2020).

[3] S. Antusch, J. Kersten, M. Lindner, M. Ratz, and M. A. Schmidt, J. High Energy Phys. 03 (2005) 024.

[4] S. Antusch, J. Kersten, M. Lindner, and M. Ratz, Nucl. Phys. B674, 401 (2003).

[5] C. M. Chen, T. Li, V. E. Mayes, and D. V. Nanopoulos, Phys. Lett. B 665, 267 (2008).

[6] C. M. Chen, T. Li, V. E. Mayes, and D. V. Nanopoulos, Phys. Rev. D 77, 125023 (2008).

[7] M. Cvetič, P. Langacker, T. Li, and T. Liu, Nucl. Phys. B709, 241 (2005).

[8] I. Esteban, M. C. Gonzalez-Garcia, A. Hernandez-Cabezudo, M. Maltoni, and T. Schwetz, J. High Energy Phys. 01 (2019) 106.

[9] T. Araki et al. (KamLAND Collaboration), Phys. Rev. Lett. 94, 081801 (2005).

[10] I. Esteban, M. C. Gonzalez-Garcia, M. Maltoni, I. MartinezSoler, and T. Schwetz, J. High Energy Phys. 01 (2017) 087.

[11] NuFit, http://www.nu-fit.org/.

[12] Y. Fukuda et al. (Super-Kamiokande Collaboration), Phys. Rev. Lett. 81, 1158 (1998); 81, 4279(E) (1998).

[13] S. Vagnozzi, E. Giusarma, O. Mena, K. Freese, M. Gerbino, S. Ho, and M. Lattanzi, Phys. Rev. D 96, 123503 (2017).

[14] E. Giusarma, M. Gerbino, O. Mena, S. Vagnozzi, S. Ho, and K. Freese, Phys. Rev. D 94, 083522 (2016).

[15] E. Giusarma, S. Vagnozzi, S. Ho, S. Ferraro, K. Freese, R. Kamen-Rubio, and K. B. Luk, Phys. Rev. D 98, 123526 (2018).

[16] S. A. Thomas, F. B. Abdalla, and O. Lahav, Phys. Rev. Lett. 105, 031301 (2010).

[17] P. A. R. Ade et al. (Planck Collaboration), Astron. Astrophys. 571, A16 (2014).
[18] R. A. Battye and A. Moss, Phys. Rev. Lett. 112, 051303 (2014).

[19] F. P. An et al. (Daya Bay Collaboration), Phys. Rev. Lett. 108, 171803 (2012).

[20] Y. Abe et al. (Double Chooz Collaboration), Phys. Rev. Lett. 108, 131801 (2012).

[21] J. K. Ahn et al. (RENO Collaboration), Phys. Rev. Lett. 108, 191802 (2012).

[22] E. Ma, Phys. Rev. D 73, 057304 (2006).

[23] E. Ma, Phys. Lett. B 660, 505 (2008).

[24] A. M. Uranga, Classical Quantum Gravity 20, S373 (2003).

[25] R. Blumenhagen, M. Cvetič, P. Langacker, and G. Shiu, Annu. Rev. Nucl. Part. Sci. 55, 71 (2005).

[26] R. Blumenhagen, B. Körs, D. Lüst, and S. Stieberger, Phys. Rep. 445, 1 (2007).

[27] F. Marchesano, Fortschr. Phys. 55, 491 (2007).

[28] M. R. Douglas and G. Moore, arXiv:hep-th/9603167.

[29] M. Berkooz, M. R. Douglas, and R. G. Leigh, Nucl. Phys. B480, 265 (1996).

[30] G. Aldazabal, S. Franco, L. E. Ibáñez, R. Rabadán, and A. M. Uranga, J. Math. Phys. (N.Y.) 42, 3103 (2001); J. High Energy Phys. 02 (2001) 047.

[31] C. Bachas, arXiv:hep-th/9503030.

[32] R. Blumenhagen, L. Görlich, B. Körs, and D. Lüst, J. High Energy Phys. 10 (2000) 006; R. Blumenhagen, B. Körs, and D. Lüst, J. High Energy Phys. 02 (2001) 030.

[33] C. Angelantonj, I. Antoniadis, E. Dudas, and A. Sagnotti, Phys. Lett. B 489, 223 (2000).

[34] D. Cremades, L. E. Ibáñez, and F. Marchesano, arXiv:hepph/0212064; J. High Energy Phys. 07 (2003) 038.

[35] H. Abe, K. S. Choi, T. Kobayashi, and H. Ohki, Nucl. Phys. B820, 317 (2009).

[36] M. Cvetič and I. Papadimitriou, Phys. Rev. D 67, 126006 (2003). 